\title{
Synovial Sarcoma of the Esophagus: A Case Report and Review of Literature
}

\author{
Abtin Doroudinia ${ }^{1, *}$, Mehrdad Bakhshayesh karam², Atosa Dorudinia ${ }^{3}$, \\ Payam Mehrian ${ }^{1}$, Farahnaz Agha-Hosseini ${ }^{1}$
}

1. Chronic Respiratory Diseases Research Center, National Research Institute of Tuberculosis and Lung Diseases (NRITLD), Masih Daneshvari Hospital, Shahid Beheshti University of Medical Sciences, Tehran, Iran

2. Pediatric Respiratory Diseases Research Center, National Research Institute of Tuberculosis and Lung Diseases (NRITLD) Masih Daneshvari Hospital, Shahid Beheshti University of Medical Sciences, Tehran, Iran

3. Tracheal Diseases Research Center (TDRC), National Research Institute of Tuberculosis and Lung Diseases (NRITLD), Masih Daneshvari Hospital. Shahid Beheshti University of Medical Sciences, Tehran, Iran

* Corresponding Author:

Abtin Doroudinia, MD

Assistant Professor, Department of Nuclear Medicine and Molecular Imaging (PET-CT fellowship), Masih Daneshvari Hospital, Department of Radiology/ PET-CT Unit, Niyavaran, Dar Abaad, Tehran, Iran Postal Code: 19569-44413

Telefax: + 982127122700

Email: abtin1354@gmail.com

Received: 08 Jan. 2017

Accepted: 10 Mar. 2017

\section{ABSTRACT}

Synovial sarcoma is an uncommon soft tissue tumor occurring mainly in the periarticular region of the extremities in young adults. It happens less frequently in the head and neck, mediastinum, lungs, heart, and digestive tract.

A 28-year-old man two months after total esophagectomy with final diagnosis of esophageal synovial sarcoma was referred to our Positron Emission Tomography (PET-CT) department for the evaluation of treatment response and further treatment planning. To our knowledge this case is the $11^{\text {th }}$ case of esophageal synovial sarcoma, being reported in the literature.

We presented the $11^{\text {th }}$ case of esophageal synovial sarcoma. Synovial sarcomas are very rare tumor entities, particularly in the gastrointestinal tract and are likely to be mistaken with other more common tumors such as gastrointestinal stromal tumors.

\section{KEYWORDS:}

Non-Hodgkin lymphoma, T cell lymphoma, Intestinal perforation

Please cite this paper as:

Doroudinia A, Bakhshayesh karam M, Dorudinia A, Mehrian P, Agha-Hosseini F. Synovial Sarcoma of the Esophagus: A Case Report and Review of Literature. Middle East $J$ Dig Dis 2017;9:111-113. DOI: 10.15171/mejdd.2017.60.

\section{INTRODUCTION}

Synovial sarcoma is a malignant mesenchymal tumor often involving the soft tissue of the extremities. ${ }^{1,2}$ Synovial sarcoma of the digestive tract is an exceptional tumor. To the best of our knowledge, only 10 cases of esophageal synovial sarcoma have been previously reported in the literature. ${ }^{3}$

\section{CASE REPORT}

A 28-year-old man initially presented with dysphagia for 6 months in addition to weight loss and other symptoms including intermittent odynophagia and generalized weakness. Physical examination showed no masses in the chest, abdomen, or extremities. Subsequently upper gastrointestinal endoscopy showed a partially obstructing, 10-cm, fusiform mass in the upper to mid esophagus, with non-ulcerating overlying mucosa. Two months after total esophagectomy with the final diagnosis of esophageal synovial sarcoma, the patient was referred for PET-CT. Pathological evaluation showed a $10 \times 10 \times 5 \mathrm{~cm}$ esophageal mass demonstrating biphasic morphological findings associated with areas of poor differentiation (figure 1).

Preoperative CT scan of the chest showed a large fusiform soft tissue 


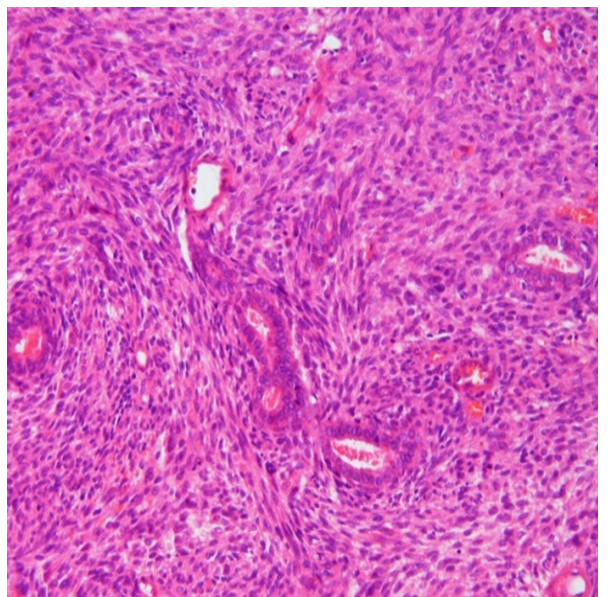

Fig.1: Synovial sarcoma; biphasic glandular structures are formed amid a spindle cell background (x200)

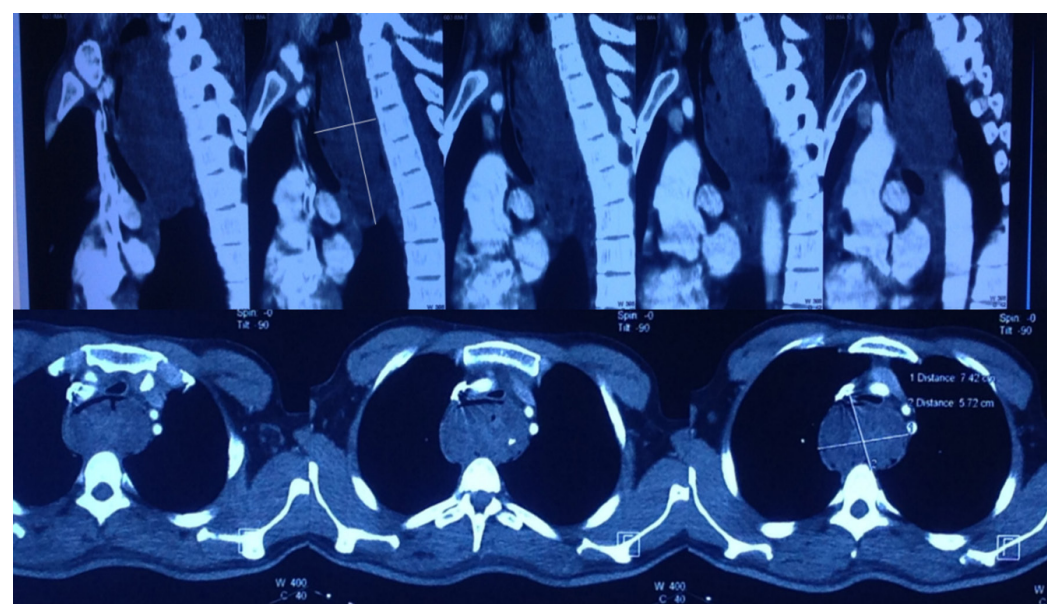

Fig.2: Preoperative chest computed tomogram with large tumor involving upper and mid esophagus.
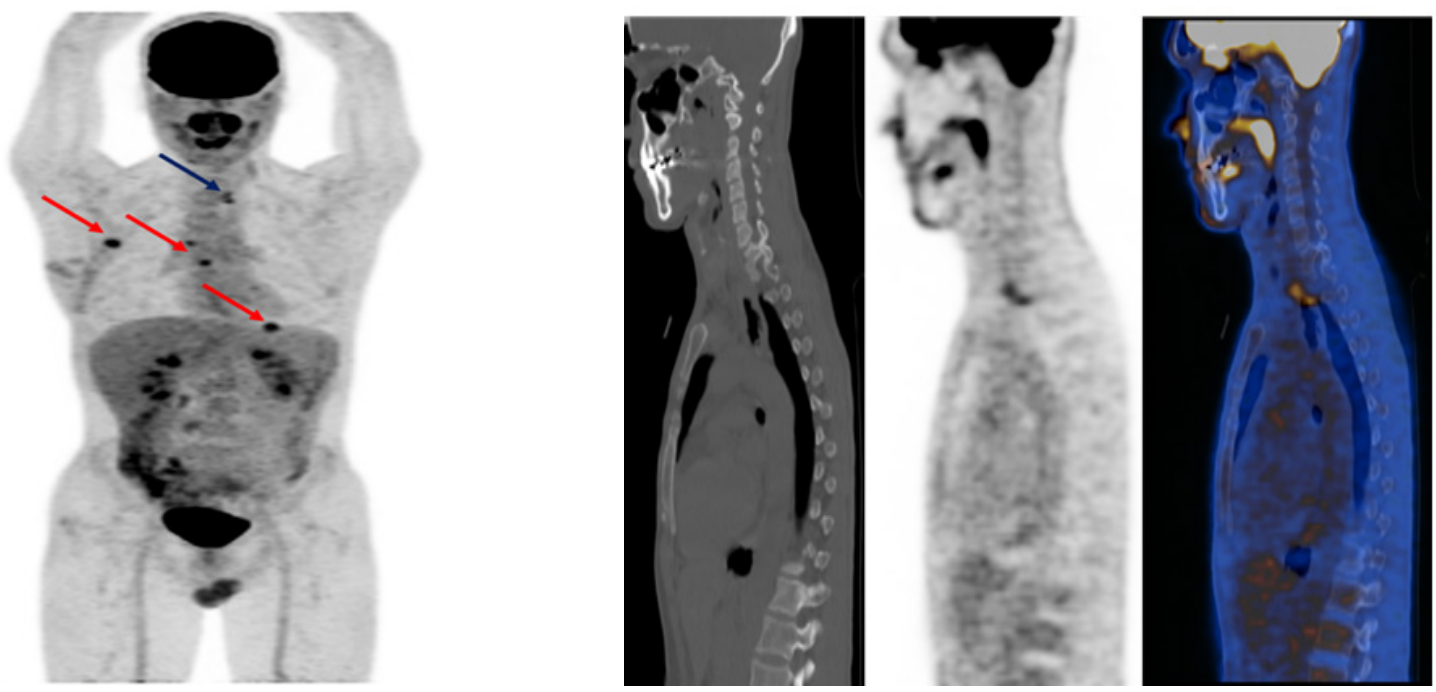

Fig.3: Postoperative PET-CT demonstrating increased metabolic activity at anastomotic site (blue arrow) and metastases (red arrows).

mass originating from the esophageal wall (figure 2). Postoperative PET-CT demonstrated postsurgical changes with mild metabolic activity at the cervical anastomotic site (figure 3, blue arrow) and evidence of metastatic tumor spread as focal increased metabolic activities in the mediastinal and hilar regions, right 5th rib, and also splenic dome (figure 3, red arrows and figure 4).

\section{DISCUSSION}

Only a few cases of esophageal synovial sarcoma have been reported. Some cases may have been previously diagnosed as sarcomatoid carcinoma or gastrointesti- nal stromal tumor (GIST). In a case series published by Alsharief and colleagues in 2012, the authors reported the first case of synovial sarcoma presenting as ileal mass. ${ }^{3}$ By reviewing the literature, there are only 10 case reports of primary esophageal synovial sarcoma in addition to one case report of gastroesophageal junction involvement. ${ }^{3}$ Another study published in 2015 by F. Sista and co-workers from Italy, only six cases of esophageal and nine cases of gastric synovial sarcoma in the literature were reported. ${ }^{4}$ Therefore to the best of our knowledge our case is the $11^{\text {th }}$ case report of esophageal synovial sarcoma based of the literature review.

Primary differential diagnosis of biphasic synovial 


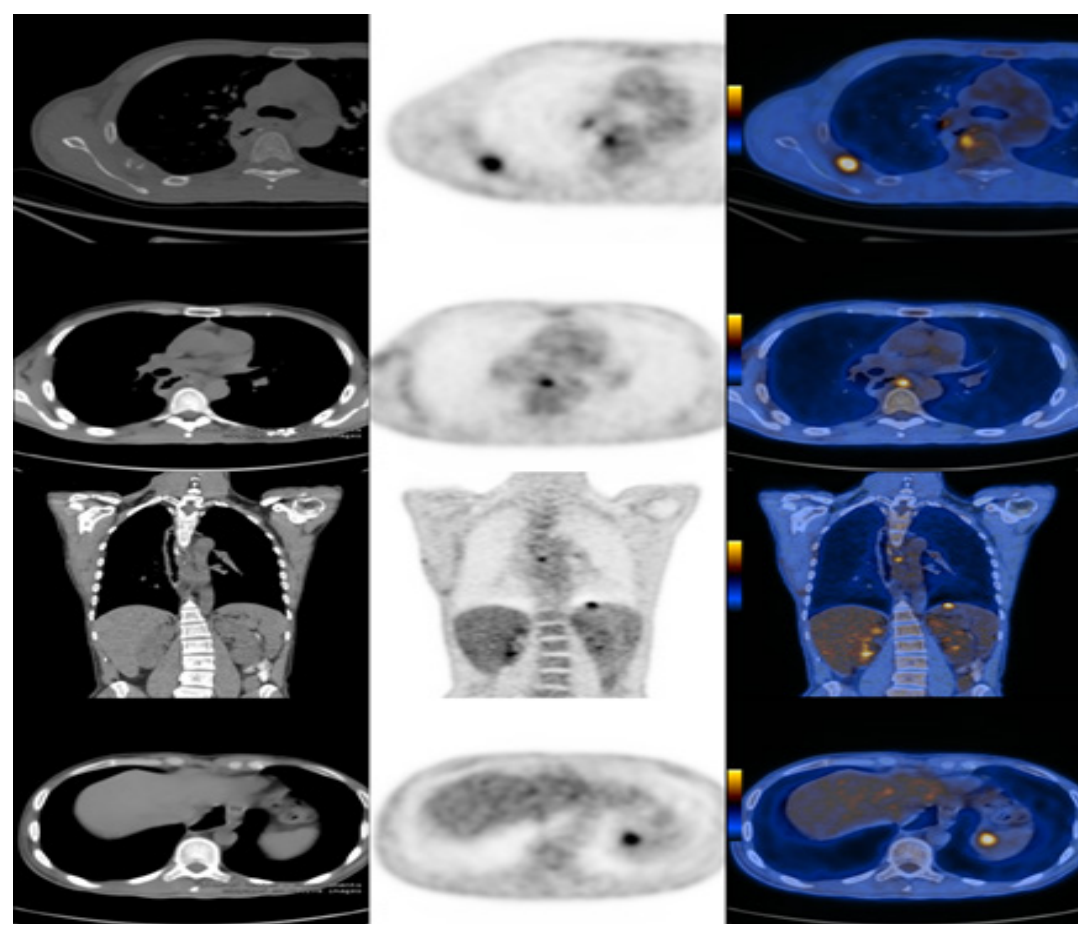

Fig.4: Metastatic lesions seen in the right fifth rib, mediastinum, and splenic dome.

sarcoma in the digestive tract is sarcomatoid carcinoma (Carcinosarcoma). On a meta-analysis performed by John PA Lau and colleagues, the authors tried to combine results across other studies to obtain a more reliable estimate of the diagnostic performance of 18F- Fluorodeoxyglucose (FDG) PET in the assessment of soft-tissue lesions. ${ }^{5}$ They concluded that 18 F-FDG PET was a useful imaging modality in the evaluation of both primary and recurrent soft-tissue sarcoma lesions with overall performance better than $\mathrm{CT}$ or magnetic resonance imaging (MRI) alone. The postoperative PET-CT findings in our case including metastatic lesions in mediastinal and hilar regions, right 5 th rib, and splenic dome have significant value for subsequent treatment strategy planning. These metastases would have been very difficult if not impossible to detect by other imaging modalities.

We presented the $11^{\text {th }}$ case of esophageal synovial sarcoma. Gastrointestinal synovial sarcomas are very rare tumors and are likely to be mistaken with other more common tumors such as GISTs.

\section{ACKNOWLEDGMENT:}

We express our special regards to technologists and technicians working in the PET-CT and Pathology Departments of Masih Daneshvari hospital.

\section{CONFLICT OF INTEREST}

The authors declare no conflict of interest related to this work.

\section{REFERENCES}

1. Palmer BV, Levene A, Shaw HJ. Synovial sarcoma of the pharynx and oesophagus. $J$ Laryngol Otol 1983;97:1173-6. doi: 10.1017/S0022215100096171.

2. Taniyama K, Sasaki N, Mukai T, Uemura N, Miyoshi N, Nakai H, et al. Carcinosarcomas of the esophagus. Pathol Int 1995;45:297-302. doi: 10.1111/ j.1442-2050.2006.00538

3. Alsharief AN, Fageeh M, Alabdulkarim Y. Monophasic synovial sarcoma presenting as a primary ileal mass: a case report and review of the literature. $J$ Med Case Reports 2012;6:83. doi: 10.1186/1752-1947-6-83

4. Sista F, Penna AD, Abruzzese V, Leardi S, Amicucci G. Intestinal Intussusception by Monophasic Synovial Sarcoma: Case Report and Literature Review. Chirurgia 2015;110: 391-395.

5. John PA, Lau J. 18F-FDG PET for the Diagnosis and Grading of Soft-Tissue Sarcoma: A Meta-Analysis. $J$ Nucl Med 2003;44:717-24. 